\title{
Early Results from HETE-2
}

N. Kawai ${ }^{1}$, A. Yoshida ${ }^{1}$, T. Tamagawa ${ }^{1}$, M. Matsuoka ${ }^{2}$, Y. Shirasaki ${ }^{2}$, G. Ricker ${ }^{3}$, G. Crew ${ }^{3}$, J.P. Doty ${ }^{3}$, A. Levine ${ }^{3}$, R. Vanderspek ${ }^{3}$, J. Villasenor ${ }^{3}$, G. Monnelly ${ }^{3}$,J.-L. Atteia ${ }^{4}$, G. Vedrenne ${ }^{4}$, J.-F. Olive ${ }^{4}$, M. Boer ${ }^{4}$, E.E. Fenimore ${ }^{5}$, M. Galassi ${ }^{5}$, J.-L. Issler ${ }^{6}$, C. Colongo ${ }^{7}$, K. Hurley ${ }^{8}$, J.G. Jernigan ${ }^{8}$, D.Q. Lamb ${ }^{9}$, C. Graziani ${ }^{9}$, G. Pizzichini ${ }^{10}$, S. Woosley ${ }^{11}$, K. Takagishi, I. Hatsukade, M. Yamauchi ${ }^{12}$, T. Cline ${ }^{13}$

${ }^{1}$ RIKEN, 2-1 Hirosawa, Wako, Saitama 351-0198, Japan,

${ }^{2}$ National Space Development Agency of Japan, Tsukuba, Japan,

${ }^{3}$ MIT Center for Space Research, Cambridge, USA,

${ }^{4}$ Centre d'Etude Spatiale des Rayonnements, Toulouse, France,

${ }^{5}$ Los Alamos National Laboratory, Los Alamos, USA,

${ }^{6}$ Centre Nationale d'Etudes Spatiales, Toulouse, France,

${ }^{7}$ Sup'Aero, Toulouse, France, ${ }^{8}$ UC-Berkeley, USA,

${ }^{9}$ University of Chicago, USA, ${ }^{10}$ TESRE/CNR, Bologna, Italy,

${ }^{11}$ UC-Santa Cruz, USA, ${ }^{12}$ Miyazaki University, Japan,

${ }^{13} N A S A$ GSFC, USA

\begin{abstract}
The High Energy Transient Explorer 2 is a small scientific satellite designed to detect and localize gamma-ray bursts (GRBs). The coordinates of GRBs detected by HETE-2 will be distributed to interested ground-based observers within seconds of burst detection, thereby allowing detailed observations of the initial phases of GRBs. HETE-2 was launched successfully on October 9,2000 . The GRB positions will start to be delivered after a few months of the complete testing and calibration of the spacecraft system and the science instruments.
\end{abstract}

\section{Introduction}

HETE-2 is a mission to provide big possibilities for small telescopes to study the extreme physics occurring at the cosmological distances.

Gamma-ray bursts have been one of the most profound mysteries in highenergy astrophysics since their discovery in the late 1960's. Attempts to associate GRB sources with any of the known categories of celestial objects had long been unsuccessful, partly because the GRBs are short transient events, typically lasting only tens of seconds. Furthermore determination of the source position in the gamma-ray band is intrinsically difficult. In order to understand the origin of the gamma-ray bursts, the most critical observation should be the accurate determination and prompt dissemination of the position of the source. With such information, detailed studies of the counterparts in optical and other wavebands should provide the clue linking GRBs to known objects. This was the consensus reached at the "High Energy Transients" workshop at Santa Cruz (1983), and 
High Energy Transient Explorer (HETE) was conceived as the mission to achieve this goal (Woosley et al 1984).

The original HETE mission was selected as an unsolicited NASA pilot mission in 1990 with G. Ricker (MIT) as the principal investigator, joined by international partners from Japan (RIKEN) and France (CESR). The satellite was launched in November 1996, but the mission was lost due to failure of the launching rocket. The importance of the study of GRBs and the HETE mission was recognized in all the participating countries, and the funding to rebuild the HETE satellite was approved in US, Japan, and France in 1997.

A breakthrough in the study of GRBs was brought about in 1997 by the BeppoSAX X-ray astronomy satellite. Its wide-field camera provided GRB positions with sufficient accuracy ( $\sim$ several arcmin) and relatively small delay ( hours), and the discovery of X-ray afterglows of GRBs with its narrow-field instruments enabled detailed follow-up observations of afterglows in X-ray, optical, and radio bands (Costa et al. 1997, van Paradijs et al. 1997, Frail et al. 1997). These observations led to identification of GRBs with sources at cosmological distances, and provided information about their distance, host galaxies, and the environment (e.g. Metzger et al. 1997). Timely localizations also started to be provided by other space missions such as RXTE ASM (Takeshima et al. 1998) and a network of Interplanetary spacecrafts (IPN) (Cline et al. 1999).

Some of the original goals of HETE were achieved by these missions, but it was also made clear that very rapid GRB position information with sufficient accuracy is very important. It was soon found that the afterglows fade rapidly following a power-law with time. Several hours after the burst, the afterglows are typically 19th magnitude or dimmer, and detailed spectroscopy is only possible with the $8-10$ meter class large telescopes. The host galaxies are typically 23 mag or dimmer, and the red-shift measurements are also possible only with the world's largest telescopes (e.g. Sahu et al. 1997, Kulkarni et al. 1998). This constraint has limited the progress in the study of the GRB population significantly. If the position information is provided 100 times earlier, i.e. within minutes of the burst, the optical afterglow could be detected at 1015th magnitude, and 1-meter class (or even smaller) telescopes could contribute spectroscopic observations. The detection of the 9 th magnitude prompt optical emission from GRB990123 by the ROTSE experiment (Akerof et al. 1999) also imply that very rapid ( $\$ 10$ seconds) positioning can lead to observations of very bright optical counterparts, which could be spectroscopically studied with small telescopes.

The current limitation of the delay in obtaining the GRB position information is due to the delay in downlinking of the satellite data, and the time required for the analysis on the ground. HETE is designed to overcome these limitations by localizing GRBs with its on-board processors, and the positions will be immediately broadcasted to the burst-alert stations distributed around the world to provide almost continuous real-time contact.

\section{Mission Overview}

The primary goals of the HETE mission are the multiwavelength observation of gamma-ray bursts (GRBs) and the prompt distribution of precise GRB coordi- 
Table 1. Scientific Instruments of HETE-2

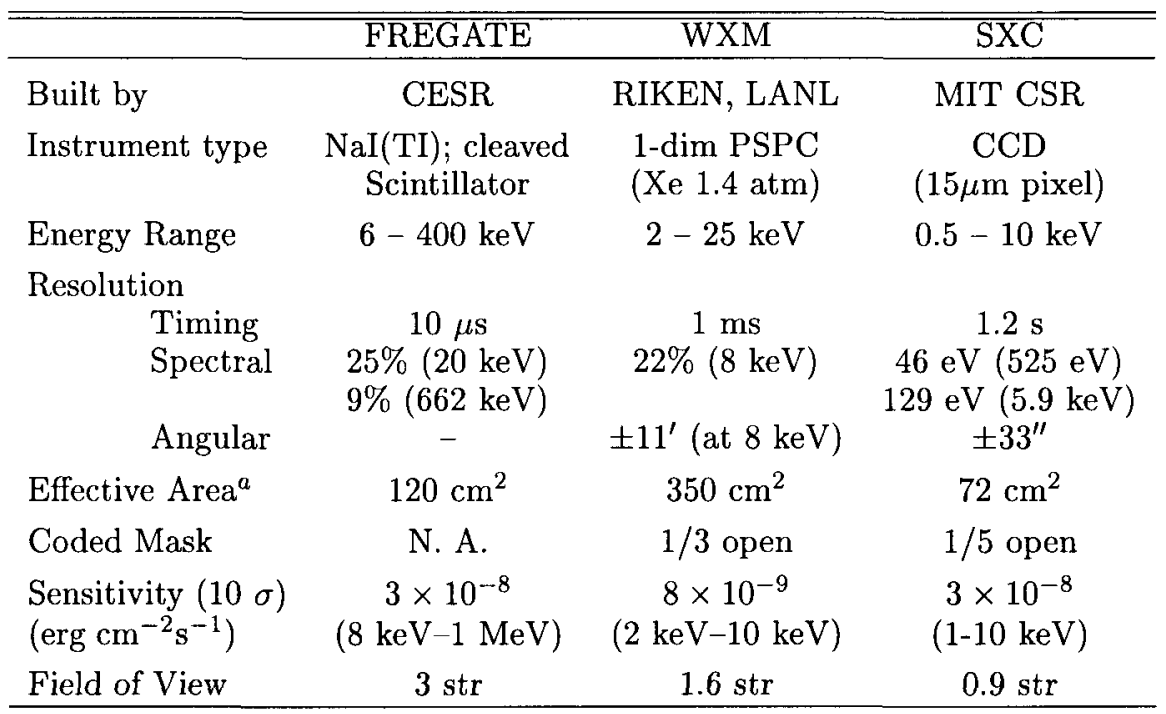

${ }^{a}$ Total effective area of the detector surface.

nates to the astronomical community for immediate follow-up observations. To achieve these goals, HETE-2 is equipped with one gamma-ray and two X-ray detectors, which share a common field of view of $\sim 1.5$ steradians, and, together, are sensitive to photons in the energy range of $0.5 \mathrm{keV}$ to over $400 \mathrm{keV}$. The two X-ray detectors are coded-aperture imagers, allowing HETE to determine the location of a GRB to a precision of 10 arc-minutes (typical) to as low as 10 arc-seconds (Table 1 ). Sophisticated on-board processing software allows the location to be calculated on board in real time, and ground post-burst analysis will provide refined localizations

In orbit, the HETE spacecraft will always point in the anti-solar direction for optimal exposure of the solar panels to the Sun. As a result, ground observers will always know approximately where HETE is observing. In addition, all bursts detected by HETE will be at least 120 degrees from the Sun and, therefore, in a prime position for observations by ground-based optical observers. The scientific instruments operate during orbit twilight and night, when the Earth is not blocking their view.

\section{Ground Stations}

The HETE-2 spacecraft communicates with the ground over either of two radio links.

- Command uplink and high-data-rate downlink occurs over the S-band, at 2.1-2.3 GHz. The downlink data rate over this link is $250 \mathrm{kbit} / \mathrm{s}$. S-band communications are done using the three Primary Ground Stations: Kwajalein Atoll (Marshall Islands), Cayenne (French Guiana), and Singapore. 
- Burst alert messages and spacecraft housekeeping data are transmitted in the VHF band, at $138 \mathrm{MHz}$. The data rate over this link is $300 \mathrm{bits} / \mathrm{s}$. VHF data reception is via the Burst Alert Station Network. There are about a dozen burst-alert stations, distributed evenly around the equator to provide continuous contact with the satellite.

All operations of the ground stations and the satellite are controlled by the MIT Command and Control Center (MCC). Commands for the spacecraft are sent to the primary ground stations from the $\mathrm{MCC}$, and data received from either the primary or burst alert stations are forwarded to the MCC.

\section{Burst Alert Network}

One of the key features of the HETE-2 satellite is its ability to calculate precise localizations of GRBs on board within seconds of burst onset, and then to transmit the burst localizations to the ground as soon as they have been calculated. The HETE-2 satellite utilizes a low-rate VHF transmitter to continuously broadcast the burst information; on the ground, an array of listen-only burst alert stations (BAS) receive the data and transmit them to the MIT Control Center. Once received at MIT, the burst information is immediately relayed to the GRB Coordinate Distribution Network (GCN) at the Goddard Space Flight Center for distribution to interested ground observers.

\subsection{Burst Information Downlinked by HETE}

Information about a GRB will come to the ground in two ways:

- The results of real-time analysis performed on the spacecraft are transmitted via the VHF to the burst alert stations. These results will be of moderate quality, as the spacecraft processors are computationally limited.

- The raw data taken by all science instruments are transmitted via the Sband data link. With these data, sophisticated ground analysis can refine the burst position.

\subsection{The GCN messages}

The GCN messages devoted to HETE data are the following:

- The HETE_ALERT message indicates that a burst has been detected. Preliminary brightness and trigger time information are included in this message, but no localization information will be available. This message is meant as a warning of an impending localization message.

- HETE_FLIGHT messages contain updates of burst intensities and durations and, most importantly, burst localizations. There can be multiple versions of this message sent during a burst: as new or better localization information becomes available, it will be relayed to the GCN. These messages are likely to be of most use to those GCN sites with automated instrumentation. 
- The HETE_FINAL message contains a summary of the best information available from analysis done on the satellite. This message should be of general interest to all.

- HETE_GND_ANALYSIS messages will be sent out after ground analysis of the raw data have been completed. More than one such message could be distributed. These messages will give useful a posteriori updates on the burst position and intensity.

\section{Mission Status}

\subsection{Launch and Orbit}

HETE-2 was launched successfully on October 9, 2000 0538(UT) with a Pegasus Rocket from the ocean near the Kwajalein Atoll of the republic of Marshall Islands at the center of the Pacific Ocean. The orbit is a semicircular low-earth orbit with an apogee of $635 \mathrm{~km}$, a perigee of $595 \mathrm{~km}$, and an inclination of $1.95^{\circ}$. The orbit life is sufficiently long to support its planned mission life of two years with possible extension of extra few years.

\subsection{Crab Calibration}

The Crab Nebula was observed by HETE-2 in November 2000 - January 2001. These data are used to calibrate spectral, timing, and astrometric performance of the detectors and the spacecraft system. The timing performance for WXM and FREGATE has been verified through the successful reconstruction of the Crab Pulsar light curve. The shadow patterns of the coded aperture were successfully recorded for hundreds of different positions of the Crab Nebula in the fields of view of WXM and SXC, which provided a data base to calibrate the alignment between the X-ray detector systems and the optical aspect cameras. All four optical cameras are fully operational. Data taken with the cameras can be used to measure the spacecraft aspect to a precision of $10-20^{\prime \prime}$ each second of orbit night. The relative positions of the cameras have been measured and thermal variations in their pointing can be measured with a precision of a few arcseconds.

\subsection{Burst Detections}

As of February 2001, in limited operations, the HETE French Gamma Telescope (FREGATE) has detected 6 cosmic GRBs (001102, 001105, 001106B, 001225, 010107, and 010126) that have been confirmed by other spacecraft, and 10 likely GRBs that have not been confirmed (001027, 001115A, 001115B, $001124,001222,001226,010111,010204,010213$, and 010225 ). The 010126 event is particularly notable as a milestone for HETE, since the event triggered the on-board burst detection software, initiating a successful VHF alert through the Burst Alert Network.

On 10 January, an unconfirmed burst event was detected by the WXM during the Crab calibration. Because of the state of the instrument calibration at the time of the burst, localization in only one direction was possible. During the FREGATE-triggered burst events of 010126 and 010204, the WXM responded by contributing appropriate data formats to the HETE downlink. However, 
because the 010126 and 010204 GRB events were outside the FOV of the WXM, localizations were not possible. Presently, the WXM is fully set to respond to triggers initiated by FREGATE.

\subsection{An Early Result: GRB010213}

On 13 February 2001 at 12:35:35 UTC, a soft spectrum, high energy transient at high galactic latitude was detected and localized by HETE. Both the WXM and the FREGATE instruments detected the event. Although the WXM and FREGATE cover extremely broad energy bands (WXM: 2-25 keV; FREGATE: 6-500 keV), the burst event was only detectable in the $2-18 \mathrm{keV}$ band by WXM, and in the 6-10 keV band by FREGATE. Because of the unusual spectrum of the transient, neither instrument triggered.

The preliminary coordinates of the burst $\left(10^{\mathrm{h}} 31^{\mathrm{m}} \cdot 36^{\mathrm{s}},+5^{\circ} 30^{\prime} \cdot 39^{\prime \prime}\right)(\mathrm{J} 2000)$, were derived by combining data from the WXM and Boresighted Optical Cameras. The statistical error radius in the WXM localization is $3.5^{\prime}$ (95\% confidence) In addition, we estimate a systematic error radius at present of $30^{\prime}$ about this location. The spacecraft aspect was known to an accuracy of $\pm 30^{\prime \prime}(95 \%$ confidence) from the optical cameras, and will be improved.

The burst exhibited a double-peaked structure and lasted about 30 seconds. The incident flux measured with the WXM (2-18 keV) at the first peak was about $0.7 \mathrm{Crab}$, and at the second peak was about 2.4 Crab. The spectrum in the $2-18 \mathrm{keV}$ range was harder than that of the Crab nebula at the first peak, and softer than that of the Crab at the second peak. The peak flux seen with FREGATE (6-10 keV) was $\sim 2$ Crab. Assuming a Crab-like spectrum, the peak energy flux was $\sim 1.510^{-8} \mathrm{erg} \mathrm{cm}^{-2} \mathrm{~s}^{-1}$ in the $6-10 \mathrm{keV}$ range.

The high galactic latitude of the source, well away from the Galactic Bulge, and the shape of its light curve suggest that it is a gamma-ray burst with an unusual spectrum. Conceivably, it could instead be a nearby X-ray burst source. A preliminary catalog search of the WXM error circle revealed no correspondence with known globular sources, cataclysmic variables, low mass X-ray binaries, or flare stars. (GCN Circular 934)

\section{References}

Akerlof, C. et al. 1999, 398, 400

Cline, T. L. 1999, A\&AS, 138, 557

Costa, E. et al. 1997, Nature, 387, 783

Frail, D. A. et al. 1997, Nature 389, 261

Kulkarni, S. R. et al. 1998 Nature, 39335

Metzger, M. R. et al. 1997, Nature, 387, 878

Sahu, K. C. et al. 1997 Nature, 387, 476

Takeshima, T. et al. 1998, in AIP Conf. Proc. 428, Gamma-Ray Bursts: 4th Huntsville Symp., eds. Meegan, Preece, \& Koshut. (New York: AIP), 414 van Paradijs, J. et al. 1997, Nature, 386, 686

Woosley, S. E. et al. 1984, in AIP Conf. Proc 115, High Energy Transients in Astrophysics., Woosley, S.E., ed. Santa Cruz, 1983. (New York: AIP) 